\title{
Management of uterine artery pseudoaneurysm: advanced ultrasonography imaging and laparoscopic surgery as an alternative method to angio-computed tomography and transarterial embolization
}

\author{
Michał Ciebiera, Aneta Słabuszewska-Jóźwiak, Kornelia Zaręba, Grzegorz Jakiel \\ First Department of Obstetrics and Gynecology, The Center of Postgraduate Medical Education, Warsaw, Poland
}

Videosurgery Miniinv 2017; 12 (1): 106-109 DOI: https://doi.org/10.5114/wiitm.2017.66503

\begin{abstract}
Uterine artery pseudoaneurysms (UAP) are rare but potentially life-threatening lesions. They may occur after traumatic deliveries, cesarean sections, and other interventions. We present a case of a 39-year-old woman with a UAP. The patient was accurately diagnosed using ultrasound imaging, with subsequent diagnostic hysteroscopy and laparoscopic excision of the UAP. In the present case, a ligation of the artery branch was performed to provide accurate hemostasis during UAP dissection. The vascular lesion was partially enucleated and removed, followed by recreation of the previous uterine shape. Power Doppler with HD flow and 3D ultrasound are accurate methods in the diagnosis of UAP. We are of the opinion that laparoscopic surgery can be on a par with transarterial embolization. During laparoscopy, the surgeon can either close the feeding vessel or remove the pathological tissue. In our opinion, this method solves the problem permanently and, after a successful case series with long-term follow-up, might be applied in other centers as well.
\end{abstract}

Key words: ultrasonography, power Doppler, uterine artery pseudoaneurysm, laparoscopy, laparoscopic surgery.

\section{Introduction}

Uterine artery pseudoaneurysms (UAP) are rare and potentially life-threatening lesions. Their actual prevalence rate remains unknown. The UAPs may occur after using an intrauterine device, traumatic delivery, pregnancy termination, cesarean section, manual placenta removal, forceps delivery, vacuum extraction, or dilatation and curettage (D\&C) [1-3]. An injury of the uterine artery wall during a traumatic procedure remains the main cause of UAP presentation. Blood dissects into peri-arterial tissues after the injury and creates a perfused sac, which communicates with the artery lumen $[3,4]$. If the blood is fully covered by the adventitia or the surrounding tissues, a pseudoaneurysm is formed. Blood fills the sac during systole and flows away during diastole [5]. The UAPs rarely reach more than $30 \mathrm{~mm}$ in diameter [6]. Their clinical symptoms vary, or they can be asymptomatic, but in some cases they may cause pelvic pain or heavy uterine bleeding $[1,5]$.

The differential diagnosis of UAPs includes acquired and congenital arteriovenous malformations (AVM), consisting of a web of mixed arteries and veins [7]. The diagnosis of UAP involves color and spectral Doppler sonography, with secondary confirmation by angiography. Angiography is considered a gold standard for the confirmation of uterine vascular lesions. The main disadvantage of computed tomography (CT) angiography is its invasive char- 
acter [5-8]. Pseudoaneurysms have a characteristic sonographic appearance, manifesting as a pulsating anechoic or hypoechoic structure connected to the parent artery by a thin vascular neck. Spectral Doppler presents turbulent arterial blood flow with a 'to and fro' pattern in UAPs $[4,5,8]$.

Arterial embolization, being a fertility-preserving treatment, is a method of choice in the management of UAP. Embolization of the uterine arteries is an effective and safe way of controlling the clinical symptoms of UAP in centers experienced in intravascular therapy. Hysterectomy is recommended for postmenopausal patients $[3,6,8,9]$. Laparoscopic methods are reported rarely [10].

\section{Case report}

We present a case of a 39-year-old woman who was admitted to the hospital after an episode of severe vaginal bleeding (the third episode in the course of 1 month). Her medical history revealed D\&C (blighted ovum) 2 years previously. On admission, speculum examination revealed a few small blood clots in the vagina. There were no signs of fresh bleeding. A pulsating enlargement of the uterine fundus was found on palpation. The red blood cell count was $3.64 \mathrm{mln} / \mu \mathrm{l}$, hemoglobin level was 9.4 $\mathrm{g} / \mathrm{dl}$, and serum $\beta$-HCG was negative.

Examination of the upper abdomen was negative. 2D transvaginal scan revealed a hypoechogenic, pulsating structure, approximately $22 \times 30 \times 23 \mathrm{~mm}$ in size, located on the left side of the uterine fundus. Color Doppler presented a swirl of colors ('yin-yang' symbol) and spectral Doppler presented a typical 'to and fro' pattern. 3D ultrasonography with power Doppler HD flow mapping was used for a more detailed examination and a connection
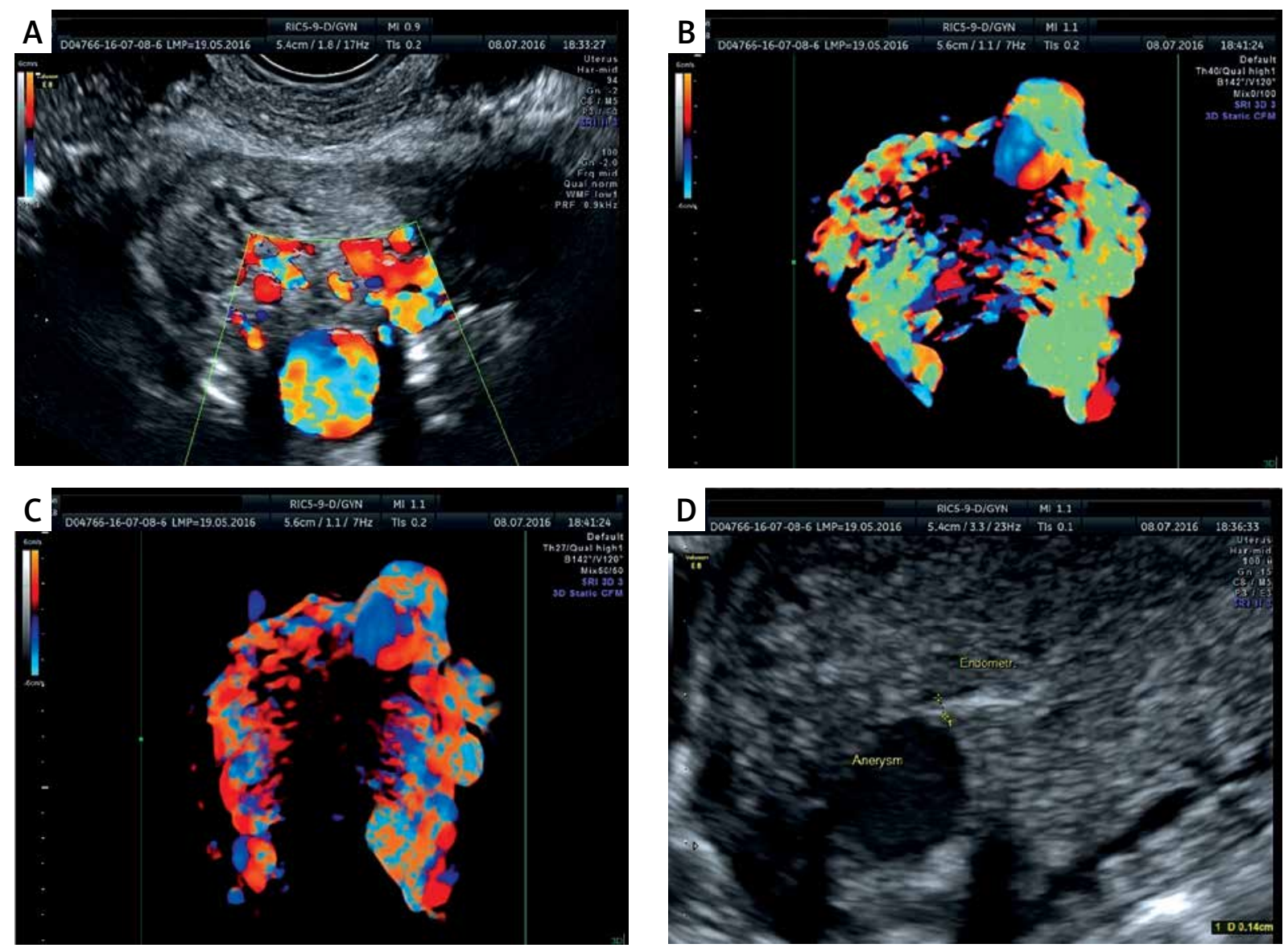

Photo 1. Uterine artery pseudoaneurysm (UAP) imaging. A - UAP in 2D with power Doppler HD flow imaging. B - UAP in 3D with power Doppler HD Flow imaging - uterine vessels. C - UAP in 3D with power Doppler HD Flow imaging - different presets. D - 2D grayscale view on the endometrium and a UAP 
between the vascular mass and the uterine artery was found (Photo 1).

Medical history and symptoms were deemed insufficient to suggest an invasive treatment. Importantly, the patient reported an allergy to iodine, so we decided to avoid CT angiography and based our diagnosis solely on the sonographic findings. The patient refused uterine artery embolization. Given her age and desire to preserve fertility, we proposed a diagnostic hysteroscopy and laparoscopic uterine artery ligation or excision of the vascular lesion.

Hysteroscopy revealed a pulsating vascular structure in the recess, just upon the uterotubal junction. We did not put the hysteroscope any further due to the high risk of heavy bleeding. The laparoscopy was performed with two $5 \mathrm{~mm}$ operational incisions and one $10 \mathrm{~mm}$ optic (umbilical) incision. Laparoscopy revealed an enlarged left side of the uterine fundus (Photo 2). After the incision of the parametrium, the ascending part of the uterine artery was partially

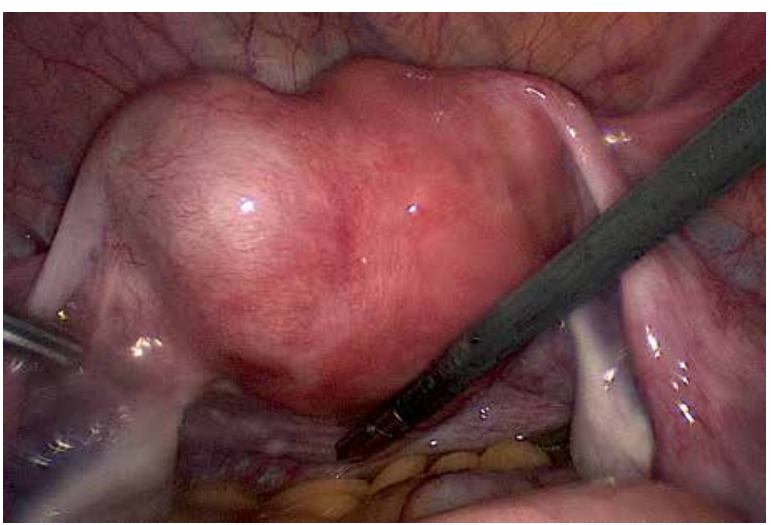

Photo 2. View of the uterine fundus during the laparoscopy

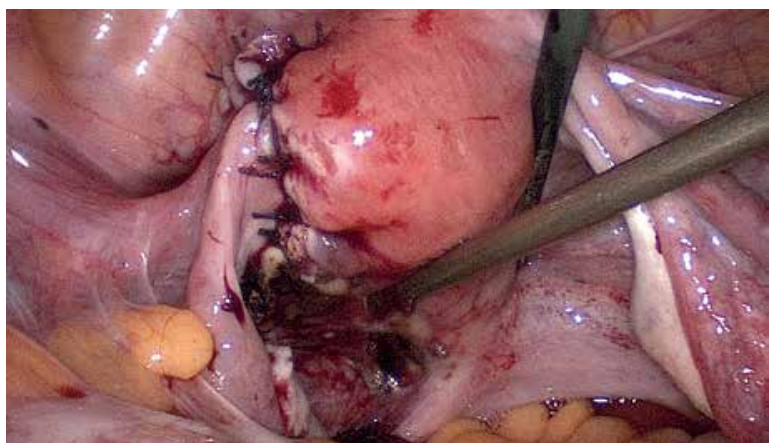

Photo 3. View of the uterus after excision of the UAP and uterine muscle suturing dissected in the direction of the change. Ligation of the artery branch was performed in the middle of its length to provide accurate hemostasis during pseudoaneurysm dissection. The uterine fundus was cut with the scissors on the left side in the expected location of the lesion. The vascular lesion was partially enucleated and then excised. The incision site was equipped with laparoscopic sutures. The previous shape of the uterus was recreated and complete hemostasis was achieved (Photo 3). Post-reconstruction hysteroscopy and chromopertubation were performed to ensure patency of both Fallopian tubes (Photo 4). No sutures were found within the uterine cavity. No complications occurred during surgery or postoperatively. Color and power Doppler sonography performed a day later presented no signs of the previously seen mass. Permanent symptom resolution was achieved, and the patient is now in the third month of follow-up.

\section{Discussion}

Due to the long interval between vessel injury and the occurrence of the clinical symptoms, the present case remains baffling. Possibly, the injury was not severe enough to cause serious bleeding and the artery wall was thinning slowly.

We are of the opinion that grayscale ultrasonography is insufficient in patients with a history of heavy vaginal bleeding. Color, spectral and power Doppler imaging should be considered in every symptomatic patient or in cases of heavy bleeding after a history of uterine surgery, etc. $[4,5,7,11]$. 3D ultrasonography can also be a valuable diagnostic method. In selected groups, ultrasonography allows for a more accurate diagnosis and earlier treatment [11].

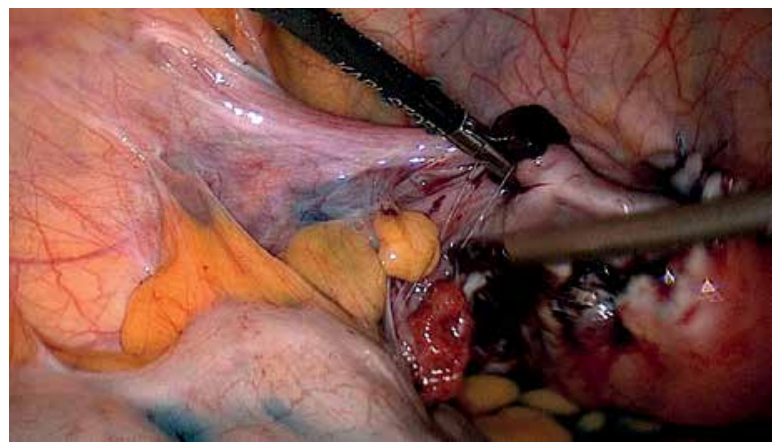

Photo 4. Patent left fallopian tube - successful chromopertubation with methylene blue 
We also believe that advanced ultrasonography can be equivalent to angio-CT in some patients.

Pseudoaneurysm is a pathology with a high risk of heavy, uncontrollable vaginal bleeding. Interventional therapy is necessary in severe bleedings, ane$\mathrm{mia}$, or in patients with further reproductive plans. Before transarterial embolization (TAE) developed and became a gold standard, hysterectomy was the most common method to treat UAP [12]. Other less radical procedures, including removal of the pseudoaneurysm, laparoscopic coagulation of the uterine artery, and unilateral ligation of the uterine artery, have been seldom reported $[5,9,10]$.

The choice of the therapeutic option depends on the risk of severe hemorrhage and the possible adverse events. Every patient needs to be fully informed about all adverse events related to the embolization procedure, especially thromboembolism of the lower extremities or formation of a vesicovaginal fistula. Therapy individualization depends on the size, shape, and clinical presentation of the pseudoaneurysm, as well as further reproductive plans of the affected patients. One of the most important predictors of further reproduction is tubal patency, which was maintained in this patient [13]. In our case, the woman refused embolization due to the fear of complications she had witnessed when her father underwent coronary angiography. We believe that laparoscopic surgery can be an alternative to embolization - pending a successful case series with a long-term follow-up [5, 6, 9, 12]. During laparoscopy, a surgeon can either close the feeding vessel or excise the pathological tissue. In our opinion, this method offers a permanent solution to the problem and may be an alternative in some cases of uterine artery pseudoaneurysm.

\section{Conflict of interest}

The authors declare no conflict of interest.

\section{References}

1. Kuwata T, Matsubara S, Kaneko Y, et al. Asymptomatic uterine artery pseudoaneurysm after cesarean section. J Obstet Gy naecol Res 2010; 36: 405-10.

2. Nagayama C, Gibo M, Nitta H, et al. Rupture of pseudoaneurysm after vaginal delivery successfully treated by selective arterial embolization. Arch Gynecol Obstet 2011; 283: 37-40.

3. Kim YA, Han YH, Jun KC, et al. Uterine artery pseudoaneurysm manifesting delayed postabortal bleeding. Fertil Steril 2008, 90: 849e11-4.
4. Abu-Yousef MM, Wiese JA, Shamma AR. The "to and fro" sign: duplex Doppler evidence of femoral artery pseudoaneurysm. Am J Roentgenol 1988; 150: 632-4.

5. Baba Y, Matsubara S, Kuwata T, et al. Uterine artery pseudoaneurysm: not a rare condition occurring after non-traumatic delivery or non-traumatic abortion. Arch Gynecol Obstet 2014; 290: 435-40.

6. Papadakos N, Wales L, Hayes K, et al. Post-traumatic pelvic pseudoaneurysm and arteriovenous fistula: combined endovascular and surgical approach. Eur J Vasc Endovasc Surg 2008; 36: 164-6.

7. Timmerman D, Wauters J, Van Calenbergh S, et al. Color Doppler imaging is a useful tool for the diagnosis and management of uterine vascular malformations. Ultrasound Obstet Gynecol 2003; 21: 570-7.

8. Bouchet P, Chabrot P, Fontarensky M, et al. Pitfalls in diagnosis of uterine artery pseudoaneurysm after Cesarean section. Ultrasound Obstet Gynecol 2012; 40: 482-3.

9. Badawy SZ, Etman A, Singh M, et al. Uterine artery embolization: the role in obstetrics and gynecology. Clin Imaging 2001; 25: 288-95.

10. Wu Y, Lin W, Yuan C, et al. Successful treatment of symptomatic arteriovenous malformation of the uterus using laparoscopic bipolar coagulation of uterine vessels. Fertil Steril 2001; 76 : 1270-11.

11. Ghi T, Giunchi S, Rossi C, et al. Three-dimensional power Doppler sonography in the diagnosis of arteriovenous malformation of the uterus. J Ultrasound Med 2005; 24: 727-31.

12. Delotte J, Chevallier P, Benoit B, et al. Pregnancy after embolization therapy for uterine arteriovenous malformation. Fertil Steril 2006; 85: e1-6.

13. Wdowiak A, Wdowiak E, Stec M, Bojar I. Post-laparoscopy predictive factors of achieving pregnancy in patients treated for infertility. Videosurgery Miniinv 2016; 11: 253-8.

Received: 16.09.2016, accepted: 29.01.2017 